\title{
3. Experimental Studies on Local Cerebral Blood Flow and Blood Gases During Acute Intracranial Hypertension
}

\author{
-With Special References to Cerebral \\ Vasoparesis- \\ Hidetaka Shida, Mitsuo Watanabe \\ and Shinken Kuramoto \\ Department of Neurosurgery, Kurume University \\ School of Medicine \\ Junichi WAKISAKA \\ Department of Ist Surgery, Kurume Universily \\ Scloot of Mcdicine
}

The state of acute intracranial hypertension especially that of the so called cerebral vasoparesis was produced experimentally and its pathologic physiology was followed, that is, the local cerebral blood flow, the EEG, and blood gases during acute intracranial hypertension were examined.

On dogs intravenous anesthesia with Nembutal was performed, and maintaining the intracranial pressure of about $300 \mathrm{mmH}_{2} \mathrm{O}$ on the average by means of epidural compression with balloon, observation was made till the death of the animals.

On impedance plethismogram, the intracranial local blood flow was estimated in the crebral cortex and in the deep brain on contralateral of the side pressuring. The EEG was also recorded with the same lead. At the same time, the arterial blood pressure, electrocardiogram, spirogram, and blood gases were also recorded or determined.

Since the intracranial pressure often declined at the beginning of increasing pressure, observation was kept up by giving pressure cach time, and one or 2 hours later it became stable at about $300 \mathrm{mmH}_{2} \mathrm{O}$.

Two or 3 hours after increasing pressure the arterial blood pressure and the intracranial pressure began spontancously to rise in parallel, giving rise to the state of cerebral vasoparesis.

After this condition lasted for about 15 to 30 minutes, both pressures swang upward rapidly. Afterwards they dropped rapidly and the animals died. Before or after this state of so-called cerebral vasoparesis oppression in breathing and circulation as well as ventilation insufficiency of blood gases appeared. The low voltage slow wave of the EEG was recorded on the one hand, and the intracranial blood flow also showed, in general, a tendency to lower on the other.

As we have seen, the pathologic physiology of acute intracanial hypertension, especially that of the so-called cerebral vasoparesis was pursued. As a result, it was found that the lasting maintenance of intracranial pressure at about 300 
$\mathrm{mmH}_{2} \mathrm{O}$ during 2 or 3 hours gives rise to the state of cerebral vasoparesis described by Moody and Mullan, and that the intracranial local blood flow still exists in spite of its general tendency to lower.

It may be assumed that oppression in breathing and circulation might greatly take part in the occurrence of so-called cerebral vasoparesis.

\title{
4. An Experimental Study on Photic Evoked Potentials in the Visual Cortex and the Brain Stem of Cats in Relation to Acute Intracranial Hypertension
}

\author{
Tadahisa Kurimoto, Kentaro Koshino, Kuniyuki Someda, \\ Satoru Kubota, Takayuki Nakajima and Naoki Kageyama \\ Department of Neurosurgery, Kansai Medical School and \\ Department of Neurosurgery, Nagoya University, \\ School of Medicine
}

The canges of photic evoked potentials associated with experimental intracranial hypertension were investigated physiologically to clarify crebral functions in 14 cats. Photic evoked potentials in the visual cortex, the brain stem and the facial nerve were recorded in sequence to time. Increased intracranial pressure, which was induced by inflation of extradural balloon, EEG and blood pressure were simultaneously recorded in relation to time.

With the increment of intracranial pressure, photic evoked potentials in the visual cortex were supressed in delayed responses with a lag of $100 \mathrm{msec}$, while those in the brain stem and the facial nerve decreased in their whole amplitudes. At the moment, EEG contained lower voltages and slower components in most of animals. Thereafter, any remarkable change of photic evoked potentials was not observed at the stages of anisocoria and following fixed dilated pupils. However, the negative waves of the photic evoked potentials in the visual cortex disappeared, reaining a positive response with a short latency associated with rapid decrease in blood pressure and flattening of EEG. On the other hand, the evoked potentials in the brain stem and the facial nerve had already disappeared.

It was interesting to note that evoked potentials in the brain stem and the facial nerve were affected earlier than those in the visual cortex.

A good recovery of evoked potentials and vital signs (pupils, blood pressure and pulse rate) was noticed in two cats in association with deflation of a balloon immediately after flattening of EEG. On the other hand, it could not be expected in cases of decompression with a lag after flattening of EEG. As far as a recovery was concerned, evoked potentials in the brain stem and the facial nerve tended to be delayed in comparison with those in the visual cortex. The animals, in which 